\title{
Endoscopic spray cryotherapy for esophageal cancer: safety and efficacy
}

\author{
Bruce D. Greenwald, MD, John A. Dumot, DO, Julian A. Abrams, MD, Charles J. Lightdale, \\ MD, Donald S. David, MD, Norman S. Nishioka, MD, Patrick Yachimski, MD, Mark H. \\ Johnston, MD, Nicholas J. Shaheen, MD, MPH, Alvin M. Zfass, MD, Jenny O. Smith, MD, \\ Kanwar Rupinder S. Gill, MD, J. Steven Burdick, MD, Damien Mallat, MD, and Herbert C. \\ Wolfsen, MD \\ Baltimore, Maryland, Cleveland, Ohio, New York, New York, Duarte, California, Boston, \\ Massachusetts, Lancaster, Pennsylvania, Chapel Hill, North Carolina, Richmond, Virginia, \\ Jacksonville, Florida, Dallas, Texas, USA
}

\section{Abstract \\ Background-Few options exist for patients with localized esophageal cancer ineligible for conventional therapies. Endoscopic spray cryotherapy with low-pressure liquid nitrogen has demonstrated efficacy in this setting in early studies. \\ Objective-To assess the safety and efficacy of cryotherapy in esophageal carcinoma. \\ Design-Multicenter, retrospective cohort study. \\ Setting-Ten academic and community medical centers between 2006 and 2009.}

Patients-Subjects with esophageal carcinoma in whom conventional therapy failed and those who refused or were ineligible for conventional therapy.

Interventions-Cryotherapy with follow-up biopsies. Treatment was complete when tumor eradication was confirmed by biopsy or when treatment was halted because of tumor progression, patient preference, or comorbid condition.

Main Outcome Measurements-Complete eradication of luminal cancer and adverse events.

Results-Seventy-nine subjects (median age 76 years, $81 \%$ male, $94 \%$ with adenocarcinoma) were treated. Tumor stage included T1-60, T2-16, and T3/4-3. Mean tumor length was $4.0 \mathrm{~cm}$ (range 1-15 cm). Previous treatment including endoscopic resection, photodynamic therapy, esophagectomy, chemotherapy, and radiation therapy failed in 53 subjects (67\%). Forty-nine completed treatment. Complete response of intraluminal disease was seen in 31 of 49 subjects (61.2\%), including 18 of 24 (75\%) with mucosal cancer. Mean (standard deviation) length of

Copyright (C) 2010 by the American Society for Gastrointestinal Endoscopy

Reprint requests: Bruce D. Greenwald, MD, Division of Gastroenterology and Hepatology, University of Maryland School of Medicine, 22 South Greene Street, Room N3W62, Baltimore, MD 21201-1595..

Current affiliations: Division of Gastroenterology and Hepatology (B.D.G.), University of Maryland School of Medicine and Greenebaum Cancer Center, Baltimore, Maryland, Department of Gastroenterology and Hepatology (J.A.D.), Cleveland Clinic, Cleveland, Ohio, Columbia University Medical Center (J.A.A., C.J.L.),NewYork,NewYork, Department of Gastroenterology (D.S.D.), City of Hope, Duarte, California, Massachusetts General Hospital and Harvard Medical School (N.S.N.), Boston, Massachusetts, Division of Gastroenterology, Hepatology, and Nutrition (P.Y.), Vanderbilt University Medical Center, Nashville, Tennessee, Lancaster Gastroenterology, Inc (M.H.J.), Lancaster, Pennsylvania, Center for Esophageal Diseases and Swallowing (N.J.S.), University of North Carolina School of Medicine, Chapel Hill, North Carolina, Virginia Commonwealth University (A.M.Z., J.O.S.), Richmond, Virginia, Sutter Gould Medical Foundation (K.R.S.G.), Modesto, California,MayoClinic Jacksonville (H.C.W.), Jacksonville, Florida, Texas Digestive Health Consultants (J.S.B.), Dallas, Texas, Digestive Health Associates of Texas (D.M.), Dallas, Texas.

Abbreviations: IQR, interquartile range; PDT, photodynamic therapy; SD, standard deviation. 
follow-up after treatment was 10.6 (8.4) months overall and 11.5 (2.8) months for T1 disease. No serious adverse events were reported. Benign stricture developed in $10(13 \%)$, with esophageal narrowing from previous endoscopic resection, radiotherapy, or photodynamic therapy noted in 9 of 10 subjects.

Limitations-Retrospective study design, short follow-up.

Conclusions-Spray cryotherapy is safe and well tolerated for esophageal cancer. Short-term results suggest that it is effective in those who could not receive conventional treatment, especially for those with mucosal cancer.

Esophagectomy traditionally has been considered standard therapy for early-stage esophageal cancer. Because of age or coexisting medical conditions, many patients are poor candidates for this procedure, and alternative treatments have been sought. Endoscopic management, particularly endoscopic resection, has been studied extensively and its effectiveness demonstrated in selected patients. ${ }^{1}$ Complete endoscopic resection of tumors covering larger areas is problematic, and repeat resection or ablation is necessary for cancer or dysplasia involving cautery margins to minimize the risk of relapse. ${ }^{2}$ Endoscopic resection is also difficult in the presence of an ulcerating tumor or in the setting of significant fibrosis or stenosis. In more advanced disease, with extension through the esophageal wall or with lymph node metastasis, initial treatment commonly consists of concurrent chemotherapy and external beam radiotherapy. Residual disease in the esophagus after chemotherapy, surgery, and/or radiotherapy in the patient not suitable for esophagectomy presents another management challenge. A significant need exists for alternative approaches in these patients.

Endoscopic spray cryotherapy using low-pressure liquid nitrogen was first reported by Johnston et $\mathrm{al}^{3}$ in the treatment of Barrett's esophagus. After their initial report, this group also described a patient with recurrent squamous cell esophageal cancer successfully treated with this modality. ${ }^{4}$ Recent single-site studies have demonstrated the safety and efficacy of cryotherapy in treating dysplasia and cancer of the esophagus. ${ }^{5,6}$ In this study, we describe the results of a multicenter study evaluating spray cryotherapy for the treatment of esophageal cancer not amenable to more traditional methods.

\section{METHODS}

\section{Subjects}

Patients treated with low-pressure liquid nitrogen endoscopic spray cryotherapy (CryoSpray Ablation System, CSA Medical, Baltimore, Md) at 10 institutions were enrolled. Site data were collected retrospectively. Eligible patients included those with esophageal carcinoma, either adenocarcinoma or squamous cell carcinoma, who received 1 or more treatments with endoscopic spray cryotherapy with either curative or palliative intent. Tumors were staged by EUS and/or endoscopic resection. The goal of treatment was elimination of all endoscopically evident tumor. All patients with mucosal carcinoma either were deemed inoperable based on medical conditions or refused esophagectomy. Patients with more invasive cancer either were deemed inoperable based on medical conditions or refused esophagectomy, had refused or were ineligible for systemic therapy including chemotherapy and radiation therapy after oncology evaluation, or systemic therapy including chemotherapy and radiation therapy had failed. Patients who previously had undergone endoscopic resection were confirmed to have residual cancer for inclusion in the study. Efficacy, but not full safety, data on 7 patients in this cohort were previously reported. 5,6 


\section{Treatment}

Informed consent was obtained from all patients. Patients were prepared for EGD in the usual fashion with an overnight fast. Sips of clear liquids with required medications were allowed up to 2 hours before the procedure. Moderate sedation with intravenous meperidine or fentanyl and midazolam or monitored anesthesia care with propofol was administered based on physician preference. EGD by using a standard upper endoscope was performed with monitoring of pulse oximetry and blood pressure. A 16F modified orogastric tube (cryodecompression tube) was passed over a guidewire through the mouth into the stomach before initiation of cryospray. This tube contains decompression ports spanning the distal 12 inches to allow gas venting of both the stomach and the esophagus during treatment. The tube was connected to continuous suction throughout the cryotherapy procedure.

Spray cryotherapy was performed with low-pressure liquid nitrogen $(<5 \mathrm{psi})$ with energy delivery of $25 \mathrm{~W}$. After passing the cryotherapy catheter through the working channel of the endoscope, liquid nitrogen was sprayed through the catheter, forming a white frost and freezing the adjacent mucosa (Fig. 1). Each mucosal site typically was frozen for 20 seconds for 3 cycles, with at least 45 seconds between freezes to allow complete tissue thawing, as verified by a return to baseline color of the tissue and complete disappearance of ice crystals after reperfusion. In situations in which the decompression tube blocked the targeted treatment site, the endoscope tip was used to gently reposition the tube out of the way, allowing appropriate treatment. One to 5 sites in the esophagus were typically treated at each session. Patients were monitored post-procedure in the usual manner and were discharged the same day. Procedures were repeated every 4 to 6 weeks. Treatment was deemed complete by the investigator after complete local tumor eradication as determined by the endoscopic appearance (Fig. 2) with endoscopic biopsy confirmation of the lack of residual disease (complete response) or when treatment was halted because of tumor progression, patient preference, or a comorbid condition precluding further endoscopy (treatment failure). In some cases, cryotherapy was continued after tumor ablation to achieve eradication of dysplasia or residual intestinal metaplasia. Follow-up endoscopy with esophageal biopsy at the treatment site was performed every 3 to 6 months after ablation was complete.

\section{Data collection and analysis}

The data for the following variables were collected for each subject: age, sex, ethnicity, histology, tumor $\mathrm{T}$ and $\mathrm{N}$ stages, previous or concurrent treatments, adverse events, number of treatment sessions, and results of cryotherapy. The cohort was divided into 2 groups for analysis. All subjects and treatments were assessed for the safety analysis. The outcomes assessed for the safety analysis included strictures, bleeding, chest pain requiring narcotics, hemorrhage, perforation, and other serious adverse events. The efficacy analysis included only those subjects who completed treatment (as judged by the treating physician, described previously). Dropouts and deaths were included in the efficacy analysis as treatment failures. The primary outcome variable of the efficacy analysis was complete eradication of all luminal cancer, confirmed by histological examination of biopsy specimens from the treated area. Secondary outcomes included complete eradication of all intestinal metaplasia. Mean and standard deviation (SD) or mean and interquartile range (IQR) are reported for continuous variables. Proportions are reported for categorical variables.

This study was approved by the institutional review board at each site.

\section{RESULTS}

Seventy-nine subjects were enrolled at the participating institutions, with 49 of them eligible for efficacy analysis (Table 1). No patients were lost to follow-up. Median age was 76 years 
(range 51-93 years, IQR 17), 81\% were male, and the histology finding was adenocarcinoma in $94 \%$ of patients. The mean (SD) tumor length treated was $4.0(3.4) \mathrm{cm}$, with the longest segment measuring $15 \mathrm{~cm}$. The median number of treatments per subject was 3 (range 1-25, IQR 3). Tumor stage included T1-60 (76\%), T2-16 (20\%), and T3/T4-3 $(4 \%)$. Characteristics were similar between the safety and efficacy cohorts. Fifty-three (67\%) subjects had received previous therapy, and 18 (24\%) received concurrent therapy for their cancer (Table 2). Endoscopic resection was the most common treatment, but other luminal therapies including photodynamic therapy (PDT), argon plasma coagulation, and radiofrequency ablation were also used. Systemic therapies included concurrent or sequential chemotherapy and external beam radiation therapy or either of these modalities alone. Four subjects who had previously refused surgery ultimately underwent esophagectomy because cryotherapy failed to eradicate tumor.

\section{Safety analysis}

A total of 332 treatments were performed in the 79 enrolled subjects. No serious adverse events, including perforation and hemorrhage, were reported. Benign stricture was noted in $10(13 \%)$ subjects. All 10 had undergone previous tumor therapy, including endoscopic resection (5 subjects), external beam radiation therapy (2 subjects), PDT alone (1 subject), PDT and endoscopic resection (1 subject), and concurrent chemotherapy and external beam radiation therapy (1 subject). Five subjects received additional endoscopic therapy during or after cryotherapy was completed, including endoscopic resection (3 subjects), endoscopic resection and argon plasma coagulation (1 subject), and argon plasma coagulation alone (1 subject). Nine of the 10 subjects were noted to have narrowing of the esophageal lumen before initiation of cryotherapy. Twenty (27\%) subjects reported posttreatment pain requiring the use of narcotic analgesics.

\section{Efficacy analysis}

Forty-nine subjects were available for the efficacy analysis. The remaining 30 subjects were still receiving cryotherapy treatment at the time of data collection. Overall, $30(61.2 \%)$ of 49 subjects demonstrated a complete response with cryotherapy for luminal disease with mean (SD) follow-up after treatment of 10.6 (8.4) months (Table 3). In this cohort, T1 tumors were present in $36(73.5 \%)$ of 49 subjects, and endoscopic remission was seen in $26(72.2 \%)$ of 36 of all T1 tumors and in 18 (75\%) of 24 mucosal (T1a) tumors with mean (SD) followup of 11.5 (2.8) months. The median number of cryotherapy sessions in the complete response group was 3 (range 1-13, IQR 3) and 3 (range 1-12, IQR 5) in the treatment failure group. In the complete response group, $63 \%$ underwent other treatments before cryotherapy compared with $68 \%$ in the treatment failure group ( $p=$ not significant). Final histology after successful treatment was normal squamous mucosa in $16(53 \%)$ subjects, intestinal metaplasia in $9(30 \%)$, low-grade dysplasia in $4(13 \%)$, and high-grade dysplasia in $1(3 \%)$.

Concurrent treatments were performed in 8 subjects in this cohort. In the complete response group, this included endoscopic resection/argon plasma coagulation (1 subject), endoscopic resection alone (1 subject), and radiofrequency ablation (1 subject). In the treatment failure group, concurrent treatments included esophagectomy (4 subjects) and argon plasma coagulation (1 subject). No significant difference was seen between the complete response and treatment failure groups based on age, tumor length, or number of subjects receiving previous or concurrent therapy. If subjects who received concurrent therapies are excluded, 27 of 41 subjects $(66 \%)$ demonstrated a complete response for luminal disease. In this cohort, T1 tumors were present in $29(71 \%)$, and endoscopic remission was seen in 23 of 29 (79\%) of all T1 tumors and in 16 of $19(84 \%)$ of all mucosal (T1a) tumors. 
This study contained a limited number of subjects with T2 and T3 disease. Of the 16 subjects with stage T2, treatment was complete in 10 with 1 to 17 months of follow-up, with only 3 subjects attaining a complete response. Two subjects with T3 disease were treated in this study. Both received chemotherapy and radiation before cryotherapy, and one also underwent esophagectomy before disease recurrence developed. The cryotherapy responder required 8 treatment sessions at 4- to 6-week intervals, with biopsy 1 month after the last treatment, demonstrating the absence of luminal cancer. The 4 patients with T2/T3 disease who completed treatment and were free of luminal disease had follow-up ranging from 2 to 6 months.

Eight subjects died of disease during the study period. T stage in this group included T1-2 (T1a-1, T1b-1), T2-5, and T3-1. All subjects had received previous treatment including PDT (1 subject), radiation (2 subjects), concurrent chemoradiation (4 subjects), or PDT and chemoradiation (1 subject). They received 2 to 12 cryotherapy treatments and survived up to 12 months after cryotherapy was halted.

\section{DISCUSSION}

Endoscopic management of superficial esophageal cancer is becoming more common because of the increasing incidence of this disease and the lack of suitability of esophagectomy in the high-risk population in which it is often diagnosed. ${ }^{7,8}$ Recent studies comparing survival rates with endoscopic and surgical management have shown comparable results in both population-based studies ${ }^{9}$ and centers with specialized expertise in managing this condition. ${ }^{7,8}$ A recent decision analysis suggests that endoscopic management is costeffective as well. ${ }^{10}$

In highly selected patients, endoscopic resection can be curative for superficial cancer. However, in a recent study, only 100 of 329 patients screened met criteria for low-risk disease, which justified endoscopic resection. In this select group, treatment was very successful, with 99 of 100 patients ultimately achieving complete response. ${ }^{1}$ Complete endoscopic resection of intestinal metaplasia and superficial adenocarcinoma has demonstrated high rates of technical success, but development of strictures limits applicability in longer esophageal segments. ${ }^{11}$ Porfimer sodium PDT with or without previous endoscopic resection has been used with mixed results in this setting, with remission of cancer seen in 20 of $24(83 \%)$ using endoscopic resection followed by PDT, ${ }^{12}$ but in only 4 of $9(44 \%)$ subjects using PDT alone. ${ }^{13}$ Endoscopic resection followed by radiofrequency ablation is effective for focal cancers, ${ }^{14}$ but the superficial depth of injury with radiofrequency ablation makes it unsuitable as a primary cancer treatment.

For more advanced esophageal cancers, conventional therapies include esophagectomy or concurrent chemo-therapy and external beam radiotherapy (chemoradiation). Although the success rate for esophagectomy in stage 1 disease is high and the mortality rate lower than commonly quoted, ${ }^{15}$ the morbidity and altered quality of life with this surgery have driven the search for alternative therapies. Concurrent chemoradiation is widely used before esophagectomy (neoadjuvant therapy) in advanced disease and as definitive therapy in those deemed to be poor surgical candidates. ${ }^{16}$ Pathological complete response to therapy is reported to be as high as $40 \%,{ }^{17}$ with 2-year local control rates between $41 \%$ and $57 \%{ }^{18,19}$ Success has been reported with salvage esophagectomy in those with local persistence or recurrence, but it is associated with increased mortality and complications, including anastomotic leak. ${ }^{20}$

Endoscopic salvage therapies for local control include PDT and endoscopic resection. In one study, complete response was seen in 8 of 13 patients treated with PDT after definitive 
combined chemoradiation failed, with 6 patients disease free at 12 months. ${ }^{21}$ Complications were common in this study, including 1 fatal tracheoesophageal fistula, 1 patient with mediastinitis, and 3 patients with strictures requiring dilation. In another series, complete response was seen in 8 of 15 patients (53\%) receiving salvage PDT. Complications developed in 7 of 15 (47\%) compared with $8 \%$ in those receiving PDT as primary therapy. These complications included fatal esophageal perforation in 2 subjects and esophageal stricture requiring dilation in $5(33.3 \%){ }^{22}$

In selected patients, endoscopic resection after definitive chemoradiation has been shown to control local disease. In one study, 16 of 93 patients with residual cancer after chemoradiation had disease limited to the mucosa/submucosa and underwent salvage endoscopic resection. Complete response was seen in half, with a 3-year survival rate of $56 \%$ compared with $38 \%$ in the total group of 93 patients. ${ }^{23}$ In a similar study, 8 (38\%) of 21 patients were alive without disease recurrence, with a 5-year overall survival rate of $49 \% .{ }^{24}$ Endoscopic resection was limited to patients with disease confined to the mucosa and submucosa and without deep ulceration. No serious adverse events were reported in either study, although bleeding, stricture, and perforation have been reported in other studies using this modality in early cancer. ${ }^{11,25}$

Endoscopic spray cryotherapy with low-pressure liquid nitrogen is a novel method for the treatment of malignant and premalignant conditions of the esophagus. It is designed to deliver a high rate of thermal energy transfer via a $7 \mathrm{~F}$ catheter in a noncontact manner. This form of cryo-therapy delivers approximately $25 \mathrm{~W}$ (joules per second) of energy to the targeted area, approximating the energy transfer of a laser and many times the energy transfer associated with cryotherapy using a standard cryotherapy probe. ${ }^{26}$ This rapid delivery of thermal energy by using liquid nitrogen at $-196^{\circ} \mathrm{C}$ effectively snap-freezes the tissue and results in immediate cell death. ${ }^{27}$ Although the degree of cell death is similar to those of the heat-related thermal modalities such as radiofrequency ablation and argon plasma coagulation, spray cryotherapy preserves the underlying tissue architecture and extracellular matrix, producing a favorable wound response with little evidence of long-term scarring. ${ }^{28}$ Spray cryotherapy is currently being used to treat a variety of different disease processes including glottic and subglottic stenosis, ${ }^{29}$ malignancy in the pleura, ${ }^{30,31}$ and radiation proctitis. ${ }^{32,33}$

In 2007, Cash et al ${ }^{4}$ reported the first use of spray cryotherapy in the palliation of recurrent esophageal squamous cell carcinoma 3 years after definitive chemoradiation. After 2 treatment sessions, the patient remained free of disease at 2-year follow-up. A recent study evaluating the safety and efficacy of spray cryotherapy included 7 patients with superficial esophageal cancer, and elimination of cancer in the esophageal lumen was seen in all 7 with follow-up ranging from 3 to 18 months. ${ }^{6}$ A recent case series of 31 patients included 5 with intramucosal carcinoma, with response to therapy seen in 4 of 5 and no cancer recurrence seen in 3 of $5 .^{5}$

In this study, we evaluated 79 cryotherapy patients from 10 institutions with esophageal carcinoma in whom other therapy failed or who refused or were ineligible for any other therapy. Because of the concern for disease extension beyond the mucosa and the potential for lymph node metastases, all of these subjects were considered for conventional therapy. Subjects entered therapy with the express goal of tumor palliation; however, endoscopic complete response was achieved in $61.2 \%$ overall and $75 \%$ of mucosal cancers with a mean follow-up of almost 1 year in the T1 group. These percentages cannot be construed as cure rates because follow-up was short and because more distant disease could not be excluded endoscopically and was not excluded systematically in this retrospective study. However, they are reasonable short-term estimates of the technology's ability to gain local control of 
the disease. The cause of treatment failure in some subjects is unknown but may have been because of insufficient treatment dose or cellular resistance to freezing. The stricture rate seen in this study was comparable to that of a previous study. ${ }^{5}$

Previous treatment with both endoscopic and systemic therapies was common, with cryotherapy being used successfully as salvage therapy. It is likely that multimodality therapy will become increasingly common in patients such as these, given the proliferation of techniques in therapeutic upper endoscopy. Cryotherapy, sometimes in combination with endoscopic resection, may offer both local control of the most significant disease and treatment of the underlying field of dysplastic or metaplastic tissue. Cryotherapy may also be useful in debulking advanced tumors; however, no published data are available for this indication.

This is the largest reported series on the use of low-pressure liquid nitrogen spray cryotherapy for the treatment of esophageal cancer. Strengths of this study include a sample drawn from both the community and academic medical centers, staging with EUS or endoscopic resection before treatment, and the use of endoscopic biopsy to confirm remission. Limitations include retrospective design and relatively short follow-up.

In summary, endoscopic spray cryotherapy is a safe and well-tolerated therapy for esophageal cancer. It was effective in achieving local control of the disease in a significant majority of subjects and demonstrated response in those with more advanced disease as well. This success was associated with no serious adverse events and a low overall complication rate. Future studies with prolonged follow-up will be useful to evaluate the durability of response.

\section{Acknowledgments}

The authors thank Mark Flasar, M.D., M.S. for statistical assistance.

DISCLOSURE: The following authors disclosed financial relationships relevant to this publication: B.D. Greenwald: Research grant from, consultant for, and advisory committee member of CSA Medical. J.A. Dumot: Research grant from and advisory committee member of CSA Medical. C.J. Lightdale: Advisory committee member of CSA Medical. N.J. Shaheen: Research grant from, consultant for, and advisory committee member of CSA Medical; research grant from BÂRRX Medical Inc; research grant from, consultant for, and speaker for Proctor \& Gamble; research grant from Oncoscope Inc; research grant from and consultant for Takeda Pharmaceuticals. All other authors disclosed no financial relationships relevant to this publication. CSA Medical supported this study through a grant for statistical analysis and by assisting in data collection at some sites. CSA Medical had no role in study design, analysis, interpretation of the data, or in writing the report.

\section{REFERENCES}

1. Ell C, May A, Pech O, et al. Curative endoscopic resection of early esophageal adenocarcinomas (Barrett's cancer). Gastrointest Endosc. 2007; 65:3-10. [PubMed: 17185072]

2. Mino-Kenudson M, Brugge WR, Puricelli WP, et al. Management of superficial Barrett's epithelium-related neoplasms by endoscopic mucosal resection: clinicopathologic analysis of 27 cases. Am J Surg Pathol. 2005; 29:680-6. [PubMed: 15832094]

3. Johnston MH, Eastone JA, Horwhat JD, et al. Cryoablation of Barrett's esophagus: a pilot study. Gastrointest Endosc. 2005; 62:842-8. [PubMed: 16301023]

4. Cash BD, Johnston LR, Johnston MH. Cryospray ablation (CSA) in the palliative treatment of squamous cell carcinoma of the esophagus. World J Surg Oncol. 2007; 5:34. [PubMed: 17367523]

5. Dumot JA, Vargo JJ 2nd, Falk GW, et al. An open-label, prospective trial of cryospray ablation for Barrett's esophagus high-grade dysplasia and early esophageal cancer in high-risk patients. Gastrointest Endosc. 2009; 70:635-44. [PubMed: 19559428] 
6. Greenwald BD, Dumot JA, Horwhat JD, et al. Safety, tolerability, and efficacy of endoscopic lowpressure liquid nitrogen spray cryotherapy in the esophagus. Dis Esophagus. 2010; 23:13-19. [PubMed: 19515183]

7. Prasad GA, Wu TT, Wigle DA, et al. Endoscopic and surgical treatment of mucosal (T1a) esophageal adenocarcinoma in Barrett's esophagus. Gastroenterology. 2009; 137:815-23. [PubMed: 19524578]

8. Schembre DB, Huang JL, Lin OS, et al. Treatment of Barrett's esophagus with early neoplasia: a comparison of endoscopic therapy and esophagectomy. Gastrointest Endosc. 2008; 67:595-601. [PubMed: 18279860]

9. Das A, Singh V, Fleischer DE, et al. A comparison of endoscopic treatment and surgery in early esophageal cancer: an analysis of surveillance epidemiology and end results data. Am J Gastroenterol. 2008; 103:1340-5. [PubMed: 18510606]

10. Pohl H, Sonnenberg A, Strobel S, et al. Endoscopic versus surgical therapy for early cancer in Barrett's esophagus: a decision analysis. Gastrointest Endosc. 2009; 70:623-31. [PubMed: 19394011]

11. Seewald S, Ang TL, Gotoda T, et al. Total endoscopic resection of Barrett esophagus. Endoscopy. 2008; 40:1016-20. [PubMed: 19065485]

12. Pacifico RJ, Wang KK, Wongkeesong LM, et al. Combined endoscopic mucosal resection and photodynamic therapy versus esophagectomy for management of early adenocarcinoma in Barrett's esophagus. Clin Gastroenterol Hepatol. 2003; 1:252-7. [PubMed: 15017665]

13. Overholt BF, Panjehpour M, Halberg DL. Photodynamic therapy for Barrett's esophagus with dysplasia and/or early stage carcinoma: long-term results. Gastrointest Endosc. 2003; 58:183-8. [PubMed: 12872083]

14. Pouw RE, Gondrie JJ, Sondermeijer CM, et al. Eradication of Barrett esophagus with early neoplasia by radiofrequency ablation, with or without endoscopic resection. J Gastrointest Surg. 2008; 12:1627-36. discussion 36-7. [PubMed: 18704598]

15. Stein HJ, Feith M, Bruecher BL, et al. Early esophageal cancer: pattern of lymphatic spread and prognostic factors for long-term survival after surgical resection. Ann Surg. 2005; 242:566-73. discussion 73-5. [PubMed: 16192817]

16. Kleinberg L, Forastiere AA. Chemoradiation in the management of esophageal cancer. J Clin Oncol. 2007; 25:4110-7. [PubMed: 17827461]

17. Tepper J, Krasna MJ, Niedzwiecki D, et al. Phase III trial of trimodality therapy with cisplatin, fluorouracil, radiotherapy, and surgery compared with surgery alone for esophageal cancer: CALGB 9781. J Clin Oncol. 2008; 26:1086-92. [PubMed: 18309943]

18. Bedenne L, Michel P, Bouche O, et al. Chemoradiation followed by surgery compared with chemoradiation alone in squamous cancer of the esophagus: FFCD 9102. J Clin Oncol. 2007; 25:1160-8. [PubMed: 17401004]

19. Stahl M, Stuschke M, Lehmann N, et al. Chemoradiation with and without surgery in patients with locally advanced squamous cell carcinoma of the esophagus. J Clin Oncol. 2005; 23:2310-7. [PubMed: 15800321]

20. Gardner-Thorpe J, Hardwick RH, Dwerryhouse SJ. Salvage oesophagectomy after local failure of definitive chemoradiotherapy. Br J Surg. 2007; 94:1059-66. [PubMed: 17657720]

21. Yano T, Muto M, Minashi K, et al. Photodynamic therapy as salvage treatment for local failures after definitive chemoradiotherapy for esophageal cancer. Gastrointest Endosc. 2005; 62:31-6. [PubMed: 15990816]

22. Lecleire S, Di Fiore F, Antonietti M, et al. Nonoperable patients with superficial esophageal cancer treated by photodynamic therapy after chemoradiotherapy have more severe complications than patients treated in primary intent. Am J Gastroenterol. 2008; 103:2215-9. [PubMed: 18844614]

23. Hattori S, Muto M, Ohtsu A, et al. EMR as salvage treatment for patients with locoregional failure of definitive chemoradiotherapy for esophageal cancer. Gastrointest Endosc. 2003; 58:65-70. [PubMed: 12838223]

24. Yano T, Muto M, Hattori S, et al. Long-term results of salvage endoscopic mucosal resection in patients with local failure after definitive chemoradiotherapy for esophageal squamous cell carcinoma. Endoscopy. 2008; 40:717-21. [PubMed: 18773340] 
25. Pech O, May A, Rabenstein T, et al. Endoscopic resection of early oesophageal cancer. Gut. 2007; 56:1625-34. [PubMed: 17938435]

26. [Accessed November 3, 2009] Percutaneous US-guided prostate cryoablation. Available at: http://www.galil-medical.com/pdfs/Prostate\%20Application\%20Sheet\%20-\%20USA $\% 202007$.pdf.

27. Krimsky WS, Broussard JN, Sarkar SA, et al. Bronchoscopic spray cryotherapy: assessment of safety and depth of airway injury. J Thorac Cardiovasc Surg. Aug 5.2009 Epub ahead of print.

28. Shepherd JP, Dawber RP. Wound healing and scarring after cryosurgery. Cryobiology. 1984; 21:157-69. [PubMed: 6713945]

29. Krimsky WS, Rodrigues M, Sarkar SA, et al. Spray cryotherapy for the treatment of glottic and subglottic stenosis. Laryngoscope. 2010; 120:473-7. Epub ahead of print. [PubMed: 20058314]

30. Downie GH, Fields E, DeKaratry D. Malignancy of the parietal pleura:novel treatment with spray cryoablation. Chest. 2009; 136(Suppl):30S-g-31S-g.

31. Downie G, Krimsky W. Response to spray cryotherapy in a patient with adenocarcinoma in the parietal pleura. Respiration. Dec 23.2009 Epub ahead of print.

32. Battish R, Shah H, Bashir R. Short-term follow-up of cryoablation treatment for radiation proctitis. Am J Gastroenterol. 2009; 104(Suppl 3):S390.

33. Shaib Y, Hou J. Complete endoscopic healing of radiation proctitis with low pressure cryoablation. Am J Gastroenterol. 2008; 103(Suppl 1):S230. 
Take-Home Message

- Effective treatments for localized esophageal cancer in those who fail or are ineligible for conventional therapies (surgery, radiation, chemotherapy, endoscopic resection) are limited. In this study, endoscopic spray cryotherapy is effective in eliminating luminal disease in a majority of patients in short-term follow-up. 


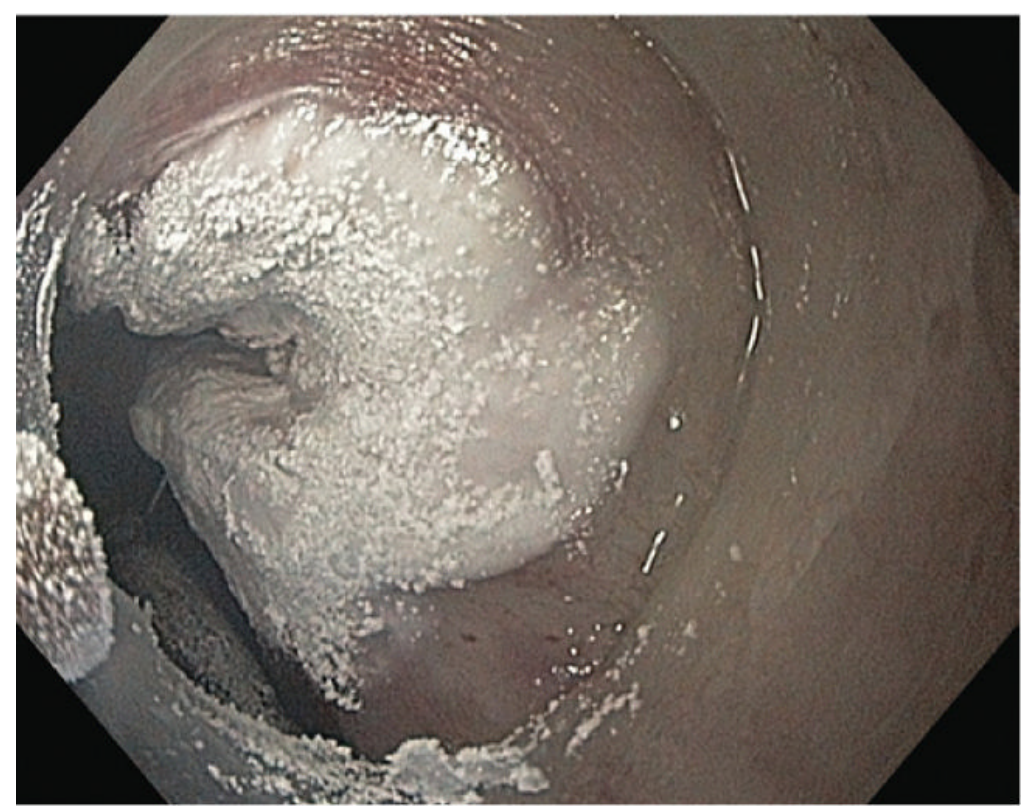

Figure 1.

Endoscopic appearance of frozen esophageal mucosa by using spray cryotherapy with liquid nitrogen. 


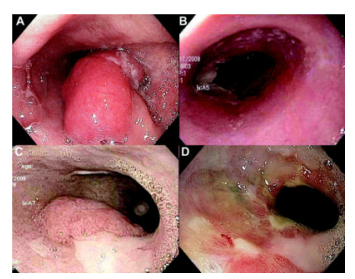

Figure 2.

Endoscopic images of a patient with persistent luminal cancer after concurrent chemotherapy and external beam radiation therapy (chemoradiation) for T2N0 esophageal adenocarcinoma. A, Initial appearance of cancer before chemoradiation. B, One month after chemoradiation, esophagitis but no cancer is detectable. $\mathbf{C}$, Appearance 5 months after chemoradiation, with adenocarcinoma and residual intestinal metaplasia present. D, After 3 cryotherapy sessions, residual cancer is no longer visible. Endoscopic biopsy specimens showed no evidence of cancer. An ink tattoo is visible marking the previous site of the tumor. 
TABLE 1

Demographics and baseline characteristics

\begin{tabular}{|c|c|c|}
\hline & Total & Completed treatment \\
\hline No. subjects & 79 & 49 \\
\hline \multicolumn{3}{|l|}{ Sex, no. $(\%)$} \\
\hline Male & $64(81.0)$ & $41(88.6)$ \\
\hline Female & $15(19.0)$ & $8(11.4)$ \\
\hline \multicolumn{3}{|l|}{ Histology, no. (\%) } \\
\hline Adenocarcinoma & $74(93.7)$ & $46(95.5)$ \\
\hline Squamous cell carcinoma & $5(6.3)$ & $3(4.5)$ \\
\hline Age, y, median (range, IQR) & $76(51-93,17)$ & $75(51-88,16)$ \\
\hline \multicolumn{3}{|l|}{ Ethnicity, no. (\%) } \\
\hline White & $72(96.0)$ & $47(95.9)$ \\
\hline African American & $2(2.7)$ & $2(4.1)$ \\
\hline Not stated & $5(1.3)$ & \\
\hline \multicolumn{3}{|l|}{ Tumor stage, no, (\%) } \\
\hline \multicolumn{3}{|l|}{$\mathrm{T} 1$} \\
\hline Mucosal & $33(41.8)$ & $24(49.0)$ \\
\hline Submucosal & $23(29.1)$ & $10(20.4)$ \\
\hline Not stated & $4(5.1)$ & $2(4.1)$ \\
\hline Total T1 & $60(76.0)$ & $36(73.5)$ \\
\hline $\mathrm{T} 2$ & $16(20.3)$ & $10(20.4)$ \\
\hline $\mathrm{T} 3$ & $2(2.5)$ & $2(4.1)$ \\
\hline $\mathrm{T} 4$ & $1(1.2)$ & $1(2.0)$ \\
\hline Tumor length, mean (SD) & $4.0(3.4)$ & $4.0(3.1)$ \\
\hline Tumor length, $\mathrm{cm}$, range & $1-15$ & $1-12$ \\
\hline No. treatments, median (range, IQR) & $3(1-25,3)$ & $3(1-13,3)$ \\
\hline
\end{tabular}

$I Q R$, Interquartile range; $S D$, standard deviation. 
TABLE 2

Therapies used in addition to spray cryotherapy

\begin{tabular}{|c|c|c|c|c|}
\hline \multirow[b]{2}{*}{ Therapy* } & \multicolumn{2}{|c|}{ Previous } & \multicolumn{2}{|c|}{ Concurren } \\
\hline & No. & $\%$ & No. & $\%$ \\
\hline Endoscopic resection & 27 & 42.2 & 9 & 45 \\
\hline Concurrent chemotherapy/external beam radiotherapy & 12 & 18.8 & & \\
\hline Photodynamic therapy & 11 & 17.2 & & \\
\hline External beam radiotherapy & 7 & 10.9 & 2 & 10 \\
\hline Concurrent chemotherapy/external beam radiotherapy, then esophagectomy & 2 & 3.1 & & \\
\hline Argon plasma coagulation & 2 & 3.1 & 3 & 15 \\
\hline Chemotherapy & 1 & 1.6 & & \\
\hline Esophageal stent & 1 & 1.6 & & \\
\hline Radiofrequency ablation & 1 & 1.6 & 2 & 10 \\
\hline Esophagectomy & & & 4 & 20 \\
\hline
\end{tabular}

* Some patients received more than 1 type of therapy. Count is the number of subjects receiving each therapy. The total number of subjects receiving previous therapy is 53 and the number receiving concurrent therapy is 18 . 
TABLE 3

Treatment results by endoscopic tumor status

\begin{tabular}{|lrrrr|}
\hline & Complete endoscopic response & \multicolumn{2}{r|}{ Persistent tumor } \\
\hline & No. & $\%$ & No. & $\%$ \\
\hline Total & 30 & 61.2 & 19 & 38.8 \\
\hline By tumor stage & & & & \\
\hline T1 & & & & \\
\hline Mucosal & 18 & 75.0 & 6 & 25.0 \\
\hline Submucosal & 6 & 60.0 & 4 & 40.0 \\
\hline Not stated & 2 & 100.0 & 0 & 0.0 \\
\hline Total T1 & 26 & 72.2 & 10 & 27.8 \\
\hline T2 & 3 & 30.0 & 7 & 70.0 \\
\hline T3 & 1 & 50.0 & 1 & 50.0 \\
\hline T4 & 0 & 0.0 & & 100.0 \\
\hline
\end{tabular}

